\title{
Management of Infection with Nontuberculosis
} Mycobacteria

\author{
Stephen H. Gillespie
}

AUTHOR:

Headings have 1. INTRODUCTION

been renum-

bered to con-

form with 1 .

1.1., 1.1.1,

style. Please

confirm re-

numbering.

The nontuberculosis mycobacteria are often naturally resistant to the conventional antibiotics and to antituberculosis drugs (1-3). In addition, providing advice for the treatment of nontuberculosis is complicated by the variable and changing designations of these organisms, the heterogeneity of the clinical syndromes and patients, and the relative lack of controlled clinical trials $(1,2)$. This chapter discusses the management of these difficult infections.

\subsection{Nomenclature}

The clinical importance of Mycobacterium tuberculosis as a major cause of death has meant that microbiologists have rightly focused on this organism. The remaining Mycobacterium species, which appeared to lack the potential to cause infection in healthy individuals, were often dismissed as "anonymous" or "atypical" (4). This approach was neither accurate nor clinically helpful. As environmental organisms, their low pathogenic potential and failure to produce diseases that resemble tuberculosis is expected. Thus, the term nontuberculosis mycobacterium (NTM) is preferred (2).

Now that conventional and molecular taxonomic techniques have been applied to this group of organisms, clinicians will increasingly be able to identify the invading mycobacteria accurately and to detect previously unrecognized species. As the pathogenic potential of each species is more accurately defined, it will become easier to choose the most appropriate drugs and management strategies.

\subsection{Epidemiology}

Mycobacteria are organisms that mainly live in the inanimate environment or as colonizers of humans and animals (5). In one sense, it is the pathogenic species $M$. tuberculosis and Mycobacterium leprae that are atypical in that they lack an environmental reservoir. Most other species of the Mycobacterium genus are found in the environment and can be isolated from soil, water, and carriage sites in animals and humans (6).

The number of cases of NTM infection has been increasing throughout the world (7). The reasons for this are varied, but are important and need to be considered to

From: Management of Multiple Drug-Resistant Infections Edited by: Stephen H. Gillespie @ Humana Press Inc., Totowa, NJ

\section{Uncorrected Proof Copy}


understand the interaction of the epidemiology of NTM and therapeutic strategies. There has been a genuine increase in the number of cases because of changes in medical practice that provide opportunities for colonization and infection, for example, colonization of intravenous long lines with rapidly growing mycobacteria (8). The increase in the number of patients who are receiving immunosuppressive therapy also provides opportunities for NTM to cause disease. The human immunodeficiency virus (HIV) epidemic brought about an enormous increase in the number of Mycobacterium aviumintracellulare cases $(9,10)$.

The number of cases has also been increasing because the diagnostic methods employed for mycobacteria have improved significantly over the last $20 \mathrm{yr}$ (11). In addition, simpler identification techniques, including molecular methods, have simplified diagnosis sufficiently that it is no longer the province of a reference laboratory. This has led to increased recognition, which has helped define the patterns of disease caused by different species and in the description of many new species (12-14).

\subsection{Scope of the Chapter}

This chapter discusses the diagnosis and management of the NTM that are difficult to treat by virtue of their natural resistance to antibiotics. The major clinical syndromes discussed include bacteremia with $M$. avium-intracellulare, cervical lymphadenopathy and pulmonary infection with NTM, and infection with rapidly growing mycobacteria.

\section{DIAGNOSIS OF NONTUBERCULOSIS MYCOBACTERIA}

The isolation of $M$. tuberculosis from a specimen is sufficient to indicate a diagnosis of tuberculosis. The only other possible explanation of this finding is crosscontamination of the specimen either in the clinical setting or in the laboratory. The diagnostic problem is more difficult for NTM. As many of these organisms can form part of the normal flora or represent environmental contaminants, a single isolate is often not sufficient to make a diagnosis $(1,2)$.

\subsection{Pulmonary Disease}

The radiological appearances of NTM pulmonary disease exhibit subtle differences from that of tuberculosis. Cavities, when present, are thin walled, and there is less surrounding infiltrate. Spread is more contiguous with more marked involvement of the pleura. Occasionally, NTM may cause a single pulmonary nodule. An important part of confirming a diagnosis of NTM infection is to exclude potential confounding diagnoses, such as tuberculosis and lung malignancy. As Mycobacterium kansasii, Mycobacterium xenopi, Mycobacterium malmoense, and the rapid growers can form part of the normal flora, multiple isolates of an NTM are required from sputum or bronchial washings obtained at different times to support a positive diagnosis (10). More weight is to be placed on single specimens that are also smear positive $(1,2)$. Alternatively, a single isolate from a biopsy specimen is diagnostic provided it is supported by compatible histology (2).

Sputum is usually an adequate specimen with which to obtain a positive diagnosis of infection with $M$. kansasii, M. xenopi, and M. malmoense. In contrast, in HIV-seronegative individuals infected with $M$. avium-intracellulare, sputum is insensitive, and a more aggressive approach using bronchial lavage should be adopted (15).

\section{Uncorrected Proof Copy}




\subsection{Lymphadenitis}

The most important part of diagnosis of lymphadenitis is to exclude tuberculosis. The diagnosis depends on granulomata in a biopsy of lymph glands in the context of a negative tuberculin test. A single isolate from a biopsy specimen is sufficient to make the diagnosis, although yields may be less than $50 \%$ of cases $(16,17)$. This in part may be because of the methods employed and the presence of fastidious mycobacterial species such as Mycobacterium haemophilum and Mycobacterium genavense.

\subsection{Cutaneous Infection}

The presence of rapidly growing mycobacteria in skin specimens must be evaluated carefully. Multiple isolates are required in clinical circumstances that support the diagnosis, for example, the presence of a plastic catheter or prosthetic device. Alternatively, individual cases may form part of known outbreaks with contaminated injections or prostheses. For Mycobacterium ulcerans and Mycobacterium marinum, the situation is often simpler as these species are likely to be isolated from patients with characteristic cutaneous lesions, making diagnosis easy. The management of these specific cutaneous infections is not discussed further.

\subsection{Disseminated Infection in HIV-Infected Individuals}

Disseminated infection with M. avium-intracellulare is usually only found in patients with advanced HIV infection who have not received antiretroviral therapy or have failed to take it. The CD count is usually low $(<50)$, and the patient has clinical signs of advanced disease (18). Patients are usually febrile and wasted and with significant anemia. Alkaline phosphatase is often elevated as hepatic involvement by M. aviumintracellulare is common. Usually, a single isolate from the blood is sufficient to confirm the diagnosis of disseminated M. avium-intracellulare infection $(1,2)$.

\subsection{Role of Susceptibility Testing}

The role of susceptibility testing in treatment choice for NTMs is controversial. There are many older studies that indicate that in vitro susceptibility test results do not correlate well with clinical outcome $(19,20)$. A more recent study of $M$. avium complex, M. malmoense, and M. xenopi found only one significant correlation of resistance and treatment failure for $M$. xenopi (21). Similarly, a study of M. avium-intracellulare infection in HIV-seronegative patients found no correlation between the in vitro susceptibility and outcome (22). However, such relationships are difficult to demonstrate unequivocally because all therapeutic regimens are with multiple drugs, and most centers only have a few patients, with the effect that these studies lack statistical power. One study of 256 patients showed a significant association $(p<0.001)$ between partial or no in vitro resistance to $1 \mathrm{mg} / \mathrm{L}$ of isoniazid and the time required for conversion of sputum from culture positive to negative, whereas complete resistance to isoniazid had a statistically significant adverse effect (23). Others have found susceptibility testing for rapid growers valuable for planning chemotherapy (24). Also, patients who responded to treatment for pulmonary $M$. avium-intracellulare received significantly more drugs to which their isolate was susceptible (25).

Much of the contradiction provided by these articles may be because mycobacterial susceptibility tests are designed for use with M. tuberculosis. NTM may be inhibited

\section{Uncorrected Proof Copy}




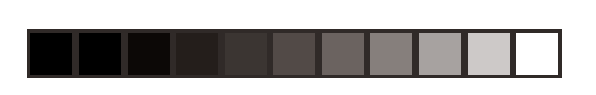

Job: Gillespie (230-4)

Chapter: 15-Gillespie

Pub Date: 1/15/04

Template: ID/7x10/Rev.05.03 (Infectious Disease)

Operator: Nettype

Date: $8 / 13 / 2003$

Revision: 1st Galleys

254

Gillespie

with antibiotic concentrations achievable in serum, but at concentrations higher than that required for tuberculosis. Thus, in vitro test results based on a breakpoint for tuberculosis will produce false resistance for an isolate of NTM $(21,22)$. The use of methods that provide minimum inhibitory concentration (MIC) data will enable regimens to be constructed specifically for NTM that take into account the achievable concentrations of antibiotics $(26,27)$.

\section{MANAGEMENT OF MYCOBACTERIUM KANSASII INFECTION}

\subsection{Pulmonary Infections}

Mycobacterium kansasii is an important pulmonary pathogen with a tendency to affect older male patients with pre-existent pulmonary disease. Mortality rates are high, but this is often because of the severe underlying conditions that coexist in these patients (28). All authorities agree that it is the inclusion of rifampicin that is responsible for favorable outcomes of culture conversion in almost all patients within 4 mo. On the other hand, resistance to this agent or its absence in the regimen underlies many of the reported examples of treatment failure $(23,29,30)$. With regimens that contain rifampicin, relapse rates are typically low, with figures of between 2.5 and $9 \%(23,28)$.

The current American Thoracic Society (ATS) recommendation for treatment of pulmonary disease caused by $M$. kansasii in adults is the regimen of isoniazid (300 $\mathrm{mg}$ ), rifampin ( $600 \mathrm{mg}$ ), and ethambutol $(25 \mathrm{mg} / \mathrm{kg}$ body weight for the first $2 \mathrm{mo}$, then $15 \mathrm{mg} / \mathrm{kg}$ body weight) given daily for $18 \mathrm{mo}$ and with at least $12 \mathrm{mo}$ of negative sputum cultures. In patients who are unable to tolerate one of these three drugs, clarithromycin would seem a reasonable alternative, but its effectiveness has not been

AUTHOR: In the paragraph "The current American," please provide
specific section instead of "see below."

AUTHOR:

Please provide specific section." established by clinical trials (see below). Pyrazinamide has no role to play in therapy for M. kansasii infections because all isolates are resistant (2).

A prospective clinical trial performed by the British Thoracic Society (BTS) in 173 patients with two sputum cultures positive for NTM showed that M. kansasii pulmonary infection responded well to 9 mo of treatment with rifampicin and ethambutol, but patients who contract this disease have a high mortality rate from other causes. Isoniazid did not appear to be a necessary part of the regimen (28). Consequently, the BTS recommend that 9 mo of rifampicin and ethambutol is adequate treatment for most patients, but when there is evidence of compromising conditions, treatment can be extended to 15-24 mo (1). The use of intermittent drug regimens or short-course therapy has not been studied sufficiently for advice to be given.

In patients who have an inadequate response or who are unable to tolerate the standard agents, prothionamide $(1 \mathrm{~g} / \mathrm{d}$ orally) and streptomycin $(0.75-1 \mathrm{~g} / \mathrm{d}$ im) could be added (1), but both are associated with frequent adverse events. Both clarithromycin and fluoroquinolones are highly active against $M$. kansasii and are likely to be beneficial $(3,31)$, although there is no clinical trial data available. These agents have proved useful in the treatment of M. avium-intracellulare infection (see below) and may be useful as part of the regimen.

Rifampicin resistance among $M$. kansasii appears to be increasing in part because of the HIV epidemic. Although rifampicin is the most important drug in the treatment of $M$. kansasii infection if patients are treated with a regimen that includes three drugs to which the infecting organism is susceptible a good outcome is likely. Many of these regimens include clarithromycin and ciprofloxacin (26).

\section{Uncorrected Proof Copy}


Job: Gillespie (230-4)

Chapter: 15-Gillespie

Pub Date: 1/15/04

Template: ID/7x10/Rev.05.03 (Infectious Disease)
Operator: Nettype

Date: $8 / 13 / 2003$

Revision: 1st Galleys

Nontuberculosis Mycobacterial Infection

\subsection{Extrapulmonary Infection}

The treatment of extrapulmonary disease should probably be similar to the pulmo-

AUTHOR: Please provide specific section." nary regimens. For lymphadenopathy, excision is recommended as this is the optimal treatment for $M$. avium-intracellulare infection, the most common cause (see below) $(1,2)$.

\section{MANAGEMENT OF MYCOBACTERIUM MALMOENSE DISEASE}

\subsection{Pulmonary Disease}

Retrospective studies have shown that patients treated for 18-24 mo with regimens that included rifampicin and ethambutol did better than those treated with other regimens or who had shorter durations of treatment (32). The addition of second- or thirdline drugs increased the rate at which adverse events were reported without improving the outcome. Surgery has an important role to play for those who are suitable for operation, and chemotherapy should be continued afterward for at least $18 \mathrm{mo}$. A clinical trial of chemotherapy in $M$. malmoense infection showed that treatment of $M$. malmoense with rifampicin and ethambutol for $2 \mathrm{yr}$ is preferable to a regimen that contains isoniazid, although there was a nonsignificant reduction in the relapse rate for the three-drug regimen. However, there was a higher death rate for the three-drug regimen (1).

Macrolides and quinolones are active in vitro (3,31), and there are some anecdotal reports of treatment response when these agents are used in the management of patients who are very susceptible to infection (33). New clinical trials have been designed and are under way to evaluate the role of macrolides and quinolones in therapeutic regimens and to detect the value of immunizing with Mycobacterium vaccae (1).

\subsection{Mycobacterium malmoense Extrapulmonary Disease}

Lymphadenitis is the most common form of M. malmoense extrapulmonary disease, and this syndrome should be treated with excision. Otherwise, extrapulmonary disease should be treated in the same way as pulmonary disease.

\section{MANAGEMENT OF MYCOBACTERIUM XENOPI DISEASE}

AUTHOR: Only 5.1. Pulmonary Disease

one secondlevel heading in this section; okay?
Mycobacterium xenopi poses many diagnostic and therapeutic problems. In some patients, M. xenopi may act as a colonizer without causing disease (34-36). Therefore, it will be present in multiple specimens, thus passing the test for "significance," although in many such cases it is not responsible for clinical symptoms. In addition, infection with $M$. xenopi is normally indolent, with disease developing over a number of years (32). Thus, an isolate in an apparently asymptomatic patient cannot be lightly dismissed, especially in HIV-infected individuals. To overcome the diagnostic difficulty, it has been proposed that the criteria for diagnosis of $M$. xenopi infection be two sputum isolations in the absence of other likely causes of symptoms (37).

Early studies have suggested that regimens should contain rifampicin and isoniazid together with ethambutol or streptomycin $(32,38)$. A clinical trial suggested that a regimen of rifampicin and ethambutol is optimum, although there is a trend to a higher cure rate when isoniazid is added, but the complication rate is increased (39). In view of the

\section{Uncorrected Proof Copy}


Job: Gillespie (230-4)

Chapter: 15-Gillespie

Pub Date: 1/15/04

Template: ID/7x10/Rev.05.03 (Infectious Disease)

256

\section{6}

Operator: Nettype

Date: $8 / 13 / 2003$

Revision: 1st Galleys

higher complication rate with added isoniazid, guidelines suggest that this drug should be included only if treatment fails to render the sputum culture negative (1).

Macrolides and quinolones may have an important role in the treatment of $M$. xenopi infections as they are active in vitro and in animal models $(3,31,40)$. There are anecdotal reports of the value of these agents (33). Clinical trial data are not yet available to inform therapeutic choice, but these could rationally be added to treatment of patients who fail to respond.

The results of medical therapy can be poor, with a 5-yr mortality of up to 57\% (although a minority of these deaths were directly attributed to mycobacterial infection) (39). Pulmonary resection is often necessary as an adjunct to treatment (41). Pulmonary resection should be considered in patients who are failing on therapy, but who otherwise have good pulmonary function and whose disease is localized. When these criteria are applied, sputum conversion is complete in all but the patients who have incomplete resection (42).

\section{MANAGEMENT OF MYCOBACTERIUM AVIUM-INTRACELLULARE DISEASE}

Infection with $M$. avium-intracellulare was once rare and was found as sporadic disease and in severely immunocompromised patients $(2,10)$. This situation was transformed by the HIV epidemic, in which disseminated M. avium-intracellulare infection and bacteremia were common late complications, usually when the CD4 count fell below 50. Management of $M$. avium-intracellulare infection is so influenced by the severity of HIV infection, it is considered separately.

AUTHOR:

There is only one second-

level heading.

Please renum ber to provide 6.2 is possible.

\subsection{HIV-Seronegative Patients}

\subsubsection{Mycobacterium avium-intracellulare Pulmonary Disease}

Pulmonary disease caused by M. avium complex in HIV-seronegative patients usually occurs in those with concomitant chronic lung disease or deficient cellular immunity, and its prevalence is increasing (10). The predisposing conditions include pneumoconiosis and silicosis because of chronic and long-term exposure to occupational dusts (e.g., from coal mining and farming) (43). For example, in one study, 73\% of patients had pre-existing pulmonary disease, 38\% smoked, and 33\% reported alcohol abuse.

The prognosis in $M$. avium complex pulmonary infections was strongly correlated with the underlying condition (44). Older studies of treatment and the natural history of disease showed that patients who are symptomatic have progressive disease that is difficult to treat, whereas many of those who were asymptomatic at the time of isolation went on to develop invasive disease (20).

Isolated pulmonary disease in otherwise healthy women has been described (45). Surveys suggest that approximately half of these patients fail therapy (15).

Treatment with three drugs, including rifampicin and isoniazid, coupled with either streptomycin or ethambutol were thought to give the best results (20). A clinical trial of treatment of M. avium-intracellulare pulmonary infection in HIV-seronegative patients suggested that the optimum treatment regimen is with rifampicin and ethambutol, and that isoniazid reduced the failure and relapse rate (39). Five-year follow-up of patients treated with this regimen showed that $15 \%$ of patients failed therapy, and 14\% relapsed (46).

\section{Uncorrected Proof Copy}


The activity of clarithromycin and quinolones suggests that they may have a role to play, and clinical trials are under way to evaluate this (39). Open trials suggested that sputum conversion rates greater than $75 \%$ can be achieved with regimens that include a macrolide (47-49). Thus, although no comparative clinical trials have yet been reported, macrolides should probably now always be included in regimens used to treat M. avium-intracellulare infections in immunocompetent patients. Intermittent therapy (three times a week) is also reported to have a similar conversion rate (50).

A recent article may give some insight into the reasons for the high relapse rate. Study of the organisms obtained from patients treated with clarithromycin who had suffered late relapses after 4 consecutive months of negative culture showed that the majority of these isolates were different from the original infecting strain. This suggests that many late relapses are caused by reinfection from this common environmental organism among patients who are highly predisposed to infection (49).

Some authorities suggest that rifabutin should be the rifamycin of choice for treatment of M. avium-intracellulare infection because of its greater in vitro activity. However, this drug has a different adverse event profile, and only comparative clinical trials can tell whether the additional activity is gained without increased adverse events.

\subsubsection{Management of Lymphadenitis}

Mycobacterium avium-intracellulare is the commonest cause of cervical lymphadenopathy in children in countries where tuberculosis has been controlled (51). Surgical excision is essential for diagnosis as the yield from fine-needle aspiration is not complete, and there is a considerable risk of sinus formation (2). Optimal treatment of this condition is surgical excision, which has a lower reoperation rate than incision and drainage, curettage, or aspiration (17). Relapse and sinus formation are rare, occurring in less than $5 \%$ of cases $(16,52)$. Antimicrobial chemotherapy appears to be unnecessary (2), although there are reports of successful management with clarithromycin monotherapy (53).

\subsubsection{Management of Disseminated Infection in HIV-Seropositive Patients}

Disseminated M. avium-intracellulare infection is a late complication of HIV infection. Since the introduction of highly active antiretroviral therapy (HAART), it has become much less common in developed countries, occurring in patients who are untreated or who have been unable to tolerate therapy. The optimal regimen has not yet been established, partly because patients with this infection are at a very late stage of HIV disease for which the clinical course is complicated by other opportunistic infections and the consequence of HIV itself. In the era of HAART, the management of disseminated $M$. avium-intracellulare infection is underwritten by therapeutic efforts to reduce the HIV viral load, increase the CD4 count, and bring about reversal of the immune deficit.

Antibiotics have an important role in reducing bacteremia, and the antibiotics able to do that include macrolides such as clarithromycin and azithromycin, quinolones such as ciprofloxacin, and rifabutin, a rifamycin. The macrolides are highly active and are the cornerstones of all regimens. They are capable of reducing the count of bacteria in the blood when given alone $(54,55)$. Monotherapy results in the rapid emergence of resistance; thus, combination therapy should be chosen.

Clinical trials have supported the superiority of clarithromycin, ethambutol, and rifabutin over rifampicin, ethambutol, clofazimine, and ciprofloxacin (56). A compara-

\section{Uncorrected Proof Copy}


tive trial suggested that lower doses of rifabutin (300 mg daily) together with ethambutol are more effective than a four-drug regimen of rifampicin, ethambutol, clofazimine, and ciprofloxacin, still retaining much of the activity of clarithromycin (1000 mg twice daily) and rifabutin (600 mg daily) doses (56).

\subsubsection{Prophylaxis of Mycobacterium avium-intracellulare}

Prophylaxis is necessary to prevent infection in patients with late-stage HIV infection with low CD4 count. Macrolides have been shown to be more effective than rifabutin, which also is an effective agent, but is associated with a higher rate of intolerance $(57,58)$. Ultimately, the choice of prophylactic agent will depend on the choice of HAART because rifabutin interacts with protease inhibitors, and patients differ considerably in their ability to tolerate drugs (59).

\section{MANAGEMENT OF INFECTION WITH RAPID GROWERS}

\subsection{Pulmonary Disease}

More than $80 \%$ of cases of pulmonary disease are caused by M. abscessus, which is the naturally most resistant member of the group of organisms (60). Treatment of $M$. abscessus infections is often disappointing. Treatment can bring about clinical improvement, but cure is rare. When surgery is technically possible, it is recommended (1). Susceptibility testing of rapidly growing mycobacteria is thought to give a good guide to treatment, and regimens should be constructed based on susceptibility test results (24). Regimens should probably include rifampicin (450 mg if the patient weighs less than $50 \mathrm{~kg}, 600 \mathrm{mg}$ if the patient weighs more than $50 \mathrm{~kg})$, ethambutol $(15 \mathrm{mg} / \mathrm{kg}$ body weight), and clarithromycin (500 mg twice daily). There are reports of the value of fluoroquinolones, sulfonamides, amikacin, cefoxitin, and penems in treatment $(1,24,61)$.

\subsection{Extrapulmonary Disease}

Many cases of infection by rapid growers occur in the context of an infected prosthetic device, for example, intravenous canullae or other implants. Successful therapy of these catheter-related infections involves removal of the catheter and antimicrobial therapy, usually for 2 to 4 mo. Although disease caused by $M$. fortuitum may resolve if the catheter is removed, reinsertion of another catheter in a similar location without drug therapy usually results in disease recurrence (as in the above case) (8). Adjunctive therapy should be with ciprofloxacin, amikacin, and clarithromycin for up to 4 mo. When there is a tunneled line that also has a tissue infection, then treatment may need to be extended for 6 mo (12).

Postinjection abscesses should be treated by surgical drainage and clarithromycin for between 3 and 6 mo. This advice comes as a result of the experience obtained from a series of outbreaks $(62,63)$.

Wound infections are one of the most common manifestations of infection with rapidly growing mycobacteria. Infections have often been associated with augmentation mammoplasty and other plastic surgery procedures $(12,64,65)$. Therapy depends of the removal of any infected foreign material, followed by 6 mo of chemotherapy $(66,67)$. Clarithromycin is the main choice, with other drugs added to prevent the emergence of resistance (68).

\section{Uncorrected Proof Copy}


Disseminated cutaneous infection is mainly with M. abscessus, usually in patients who are compromised by renal failure or corticosteroid therapy (69). This is one of the most common presentations of nonpulmonary infection with rapidly growing organisms (12). Treatment includes drainage of any abscesses coupled with clarithromycin for $6 \mathrm{mo}$ and with another agent to which the isolate is susceptible during the first $2 \mathrm{mo}(68)$.

\section{SUMMARY}

Infections with NTM continue to pose significant diagnostic and therapeutic problems for clinicians. Infection often occurs in the context of other serious disease, which may influence the outcome more than the infective process. Diagnosis can be difficult, but modern laboratory methods are improving rapidly. For several important infections, clinical trial information is helping to inform clinicians (39). The results of trials currently under way to elucidate the activity of quinolones and macrolides may soon improve the evidence base on for defining more effective regimens.

\section{REFERENCES}

1. Joint Tuberculosis Committee. Management of opportunist mycobacterial infections: Joint Tuberculosis Committee guidelines 1997. Thorax 2000; 55:210-218.

2. American Thoracic Society. Diagnosis and treatment of disease caused by nontuberculous mycobacteria. Am J Respir Crit Care Med 1997; 156:S1-S25.

3. Gillespie SH, Morrissey I, Everett D. A comparison of the bactericidal activity of quinolone antibiotics in a Mycobacterium fortuitum model. J Med Microbiol 2001; 50:565-570.

4. Runyon EH. Anonymous mycobacteria in pulmonary disease. Med Clin North Am 1957; 43:273-290.

5. O'Brien RJ, Geiter LJ, Snider DE Jr. The epidemiology of nontuberculous mycobacterial diseases in the United States. Results from a national survey. Am Rev Respir Dis 1987; 135:1007-1014.

6. Kirschner RA Jr, Parker BC, Falkinham JO III. Epidemiology of infection by nontuberculous mycobacteria. Mycobacterium avium, Mycobacterium intracellulare, and Mycobacterium scrofulaceum in acid, brown-water swamps of the southeastern United States and their association with environmental variables. Am Rev Respir Dis 1992; 145(2, pt. 1):271-275.

7. Lambden K, Watson J, Knerer G, Ryan MJ, Jenkins PA. Opportunist mycobacteria in England and Wales: 1982 to 1994. Commun Dis Rep CDR Rev 1996; 6:R147-R151.

8. McWhinney PH, Yates M, Prentice HG, Thrussell M, Gillespie SH, Kibbler CC. Infection caused by Mycobacterium chelonae: a diagnostic and therapeutic problem in the neutropenic patient. Clin Infect Dis 1992; 14:1208-1212.

9. Tortoli E, Bartoloni A, Bottger EC, et al. Burden of unidentifiable mycobacteria in a reference laboratory. J Clin Microbiol 2001; 39:4058-4065.

10. Falkinham JO III. Epidemiology of infection by nontuberculous mycobacteria. Clin Microbiol Rev 1996; 9:177-215.

11. Hale YM, Pfyffer GE, Salfinger M. Laboratory diagnosis of mycobacterial infections: new tools and lessons learned. Clin Infect Dis 2001; 33:834-846.

12. Brown-Elliott BA, Wallace RJ Jr. Clinical and taxonomic status of pathogenic nonpigmented or late-pigmenting rapidly growing mycobacteria. Clin Microbiol Rev 2002; 15:716-746.

13. Tortoli E, Bartoloni A, Erba ML, et al. Human infections due to Mycobacterium lentiflavum. J Clin Microbiol 2002; 40:728-729.

14. Turenne C, Chedore P, Wolfe J, Jamieson F, May K, Kabani A. Phenotypic and molecular AU: \# 14: characterization of clinical isolates of Mycobacterium elephantis from human specimens. Need source.

\section{Uncorrected Proof Copy}


15. Huang JH, Kao PN, Adi V, Ruoss SJ. Mycobacterium avium-intracellulare pulmonary infection in HIV-negative patients without pre-existing lung disease: diagnostic and management limitations. Chest 1999; 115:1033-1040.

16. Danielides V, Patrikakos G, Moerman M, Bonte K, Dhooge C, Vermeersch H. Diagnosis, management and surgical treatment of non-tuberculous mycobacterial head and neck infection in children. ORL J Otorhinolaryngol Relat Spec 2002; 64:284-289.

17. Flint D, Mahadevan M, Barber C, Grayson D, Small R. Cervical lymphadenitis due to nontuberculous mycobacteria: surgical treatment and review. Int J Pediatr Otorhinolaryngol 2000; 53:187-194.

18. Dautzenberg B, Olliaro P, Ruf B, et al. Rifabutin versus placebo in combination with three drugs in the treatment of non-tuberculous mycobacterial infection in patients with AIDS. Clin Infect Dis 1996; 22:705-708.

19. Pfeutze KM, Nuchprayoon CV, Berg GS, Pamintuan R. Present status of open negative cavities due to photochromogenic mycobacteria among co-operative patients. Am Rev Respir Dis 1966; 94:467-470.

20. Hunter AM, Campbell IA, Jenkins PA, Smith PA. Treatment of pulmonary infection caused by mycobacteria of Mycobacterium avium-intracellulare complex. Thorax 1981; 36:326-329.

21. Heginbothom ML. The relationship between the in vitro drug susceptibility of opportunist mycobacteria and their in vivo response to treatment. Int J Tuberc Lung Dis 2001; 5:539-545.

22. Jenkins PA, Banks J, Campbell IA, Gelder CM, Prescott RJ, Smith AP. Pulmonary disease caused by Mycobacterium avium-intracellulare in HIV sero-negative patients: a 5 year followup of patients receiving standardised treatment. Int J Tuberc Lung Dis 2002; 6:628-625.

23. Ahn CH, Lowell JR, Ahn SS, Ahn S, Hurst GA. Chemotherapy for pulmonary disease due to Mycobacterium kansasii: efficacies of some individual drugs. Rev Infect Dis 1981; 3:1028-1034.

24. Wallace RJ Jr, Swenson JM, Silcox VA, Bullen MG. Treatment of non-pulmonary infections due to Mycobacterium fortuitum and Mycobacterium chelonei on the basis of in vitro susceptibilities. J Infect Dis 1985; 152:200-214.

25. Horsburgh CR Jr, Mason UG III, Heifets LB, Southwick K, Labrecque J, Iseman MD. Response to therapy of pulmonary Mycobacterium avium-intracellulare infection correlates with results of in vitro susceptibility testing. Am Rev Respir Dis 1987; 135:418-421.

26. Wallace RJ Jr, Dunbar D, Brown BA, et al. Rifampin-resistant Mycobacterium kansasii. Clin Infect Dis 1994; 18:736-743.

27. Rastogi N, Goh KS, Guillou N, Labrousse V. Spectrum of drugs against atypical mycobacteria: how valid is the current practice of drug susceptibility testing and the choice of drugs? Zentralbl Bakteriol 1992; 277:474-484.

28. Jenkins PA, Banks J, Campbell IA, Smith AP. Mycobacterium kansasii pulmonary infection: a prospective study of the results of 9 months of treatment with rifampicin and ethambutol. Research Committee, British Thoracic Society. Thorax 1994; 49:442-445.

29. Banks J, Hunter AM, Campbell IA, Jenkins PA, Smith AP. Pulmonary infection with Mycobacterium kansasii in Wales, 1970-9: review of treatment and response. Thorax 1984; 39: 376-382.

30. Pezzia W, Raleigh JW, Bailey MC, Toth EA, Silverblatt J. Treatment of pulmonary disease due to Mycobacterium kansasii: recent experience with rifampin. Rev Infect Dis 1981; 3:1035-1039.

31. Yew WW, Piddock LJ, Li MS, Lyon D, Chan CY, Cheng AF. In-vitro activity of quinolones and macrolides against mycobacteria. J Antimicrob Chemother 1994; 34:343-351.

32. Banks J, Hunter AM, Campbell IA, Jenkins PA, Smith AP. Pulmonary infection with Mycobacterium xenopi: review of treatment and response. Thorax 1984; 39:376-382.

33. Scmitt H, Schnitzler N, Riehl J, Adam G, Siebert H-G, Haase G. Successful treatment of pulmonary Mycobacterium xenopi infection in a natural killer cell-deficient patient with clarithromycin, rifabutin, and sparfloxacin. Clin Infect Dis 1999; 29:120-124.

\section{Uncorrected Proof Copy}


34. Smith MJ, Citron KM. Clinical review of pulmonary disease caused by Mycobacterium xenopi. Thorax 1983; 38:373-377.

35. Jiva TM, Jacoby HM, Weymouth LA, Kaminski DA, Portmore AC. Mycobacterium xenopi: innocent bystander or emerging pathogen? Clin Infect Dis 1997; 24:226-232.

36. Simor AE, Salit IE, Vellend H. The role of Mycobacterium xenopi in human disease. Am Rev Respir Dis 1984; 129:435-438.

37. Juffermans NP, Verbon A, Danner SA, Kuijper EJ, Speelman P. Mycobacterium xenopi in HIV-infected patients: an emerging pathogen. AIDS 1998; 12:1661-1666.

38. Costrini AM, Mahler DA, Gross WM, Hawkins JE, Yesner R, D'Esopo ND. Clinical and roentgenographic features of nosocomial pulmonary disease due to Mycobacterium xenopi. Am Rev Respir Dis 1981; 123:104-109.

39. Research Committee BTS. First randomised trial of treatments for pulmonary disease caused by $M$. avium intracellulare, M. malmoense, and $M$. xenopi in HIV negative patients: rifampicin, ethambutol and isoniazid versus rifampicin and ethambutol. Thorax 2001; 56:167-172.

40. Lounis N, Truffot-Pernot C, Bentoucha A, Robert J, Ji B, Grosset J. Efficacies of clarithromycin regimens against Mycobacterium xenopi in mice. Antimicrob Agents Chemother 2001; 45:3229-3230.

41. Parrot RG, Grosset JH. Post-surgical outcome of 57 patients with Mycobacterium xenopi pulmonary infection. Tubercle 1988; 69:47-55.

42. Lang-Lazdunski L, Offredo C, Pimpec-Barthes F, Danel C, Dujon A, Riquet M. Pulmonary resection for Mycobacterium xenopi pulmonary infection. Ann Thorac Surg 2001; 72:1877-1882.

43. Schaefer WB, Birn KJ, Jenkins PA, Marks J. Infection with the avian-Battey group of mycobacteria in England and Wales. BMJ 1969; 2:412-415.

44. Contreras MA, Cheung OT, Sanders DE, Goldstein RS. Pulmonary infection with nontuberculous mycobacteria. Am Rev Respir Dis 1988; 137:149-152.

45. Prince DS, Peterson DD, Steiner RM, et al. Infection with Mycobacterium avium complex in patients without predisposing conditions. N Engl J Med 1989; 321:863-868.

46. Pulmonary disease caused by Mycobacterium avium-intracellulare in HIV-negative patients: 5-year follow-up of patients receiving standardised treatment. Int J Tuberc Lung Dis 2002; 6:628-634.

47. Griffith DE, Brown BA, Murphy DT, Girard WM, Couch L, Wallace RJ Jr. Initial (6month) results of three-times-weekly azithromycin in treatment regimens for Mycobacterium avium complex lung disease in human immunodeficiency virus-negative patients. J Infect Dis 1998; 178:121-126.

48. Wallace RJ Jr, Brown BA, Griffith DE, et al. Initial clarithromycin monotherapy for Mycobacterium avium-intracellulare complex lung disease. Am J Respir Crit Care Med 1994; 149:1335-1341.

49. Wallace RJ Jr, Zhang Y, Brown-Elliott BA, et al. Repeat positive cultures in Mycobacterium intracellulare lung disease after macrolide therapy represent new infections in patients with nodular bronchiectasis. J Infect Dis 2003; 186:266-273.

50. Griffith DE, Brown BA, Cegielski P, Murphy DT, Wallace RJ Jr. Early results (at 6 months) with intermittent clarithromycin-including regimens for lung disease due to Mycobacterium avium complex. Clin Infect Dis 2000; 30:288-292.

51. Grange JM, Yates MD, Pozniak A. Bacteriologically confirmed non-tuberculous mycobacterial lymphadenitis in southeast England: a recent increase in the number of cases. Arch Dis Child 1995; 72:516-517.

52. Rahal A, Abela A, Arcand PH, Quintal MC, Lebel MH, Tapiero BF. Nontuberculous mycobacterial adenitis of the head and neck in children: experience from a tertiary care pediatric center. Laryngoscope 2001; 111:1791-1796.

53. Tunkel DE, Romaneschi KB. Surgical treatment of cervicofacial nontuberculous mycobacterial adenitis in children. Laryngoscope 1995; 105:1024-1028.

\section{Uncorrected Proof Copy}


54. Dautzenberg B, Saint MT, Meyohas MC, et al. Clarithromycin and other antimicrobial agents in the treatment of disseminated Mycobacterium avium infections in patients with acquired immunodeficiency syndrome. Arch Intern Med 1993; 153:368-372.

55. Hoy J, Mijch A, Sandland M, Grayson L, Lucas R, Dwyer B. Quadruple-drug therapy for Mycobacterium avium-intracellulare bacteremia in AIDS patients. J Infect Dis 1990; 161: 801-805.

56. Shafran SD, Singer J, Zarowny DP, et al. A comparison of two regimens for the treatment of Mycobacterium avium complex bacteremia in AIDS: rifabutin, ethambutol, and clarithromycin versus rifampin, ethambutol, clofazimine, and ciprofloxacin. Canadian HIV Trials Network Protocol 010 Study Group. N Engl J Med 1996; 335:377-383.

57. Phillips P, Chan K, Hogg R, et al. Azithromycin prophylaxis for Mycobacterium avium complex during the era of highly active antiretroviral therapy: evaluation of a provincial program. Clin Infect Dis 2002; 34:371-378.

58. Havlir DV, Dube MP, Sattler FR, et al. Prophylaxis against disseminated Mycobacterium avium complex with weekly azithromycin, daily rifabutin, or both. California Collaborative Treatment Group. N Engl J Med 1996; 335:392-398.

59. Cohn SE, Kammann E, Williams P, Currier JS, Chesney MA. Association of adherence to Mycobacterium avium complex prophylaxis and antiretroviral therapy with clinical outcomes in acquired immunodeficiency syndrome. Clin Infect Dis 2002; 34:1129-1136.

60. Griffith DE, Girard WM, Wallace RJ Jr. Clinical features of pulmonary disease caused by rapidly growing mycobacteria. An analysis of 154 patients. Am Rev Respir Dis 1993; 147: 1271-1278.

61. Tanaka E, Kimoto T, Tsuyuguchi K, Suzuki K, Amitani R. Successful treatment with faropenem and clarithromycin of pulmonary Mycobacterium abscessus infection. J Infect Chemother 2002; 8:252-255.

62. Galil K, Miller LA, Yakrus MA, et al. Abscesses due to Mycobacterium abscessus linked to injection of unapproved alternative medication. Emerg Infect Dis 1999; 5:681-687.

63. Villanueva A, Calderon RV, Vargas BA, et al. Report on an outbreak of post-injection abscesses due to Mycobacterium abscessus, including management with surgery and clarithromycin therapy and comparison of strains by random amplified polymorphic DNA polymerase chain reaction. Clin Infect Dis 1997; 24:1147-1153.

64. Clegg HW, Foster MT, Sanders WE Jr, Baine WB. Infection due to organisms of the Mycobacterium fortuitum complex after augmentation mammoplasty: clinical and epidemiologic features. J Infect Dis 1983; 147:427-433.

65. Bolan G, Reingold AL, Carson LA, et al. Infections with Mycobacterium chelonei in patients receiving dialysis and using processed hemodialyzers. J Infect Dis 1985; 152: 1013-1019.

66. Morris-Jones R, Fletcher C, Morris-Jones S, Brown T, Hilton RM, Hay R. Mycobacterium abscessus: a cutaneous infection in a patient on renal replacement therapy. Clin Exp Dermatol 2001; 26:415-418.

67. Ozluer SM, De'Ambrosis BJ. Mycobacterium abscessus wound infection. Australas J Dermatol 2001; 42:26-29.

68. Wallace RJ Jr, Tanner D, Brennan PJ, Brown BA. Clinical trial of clarithromycin for cutaneous (disseminated) infection due to Mycobacterium chelonae. Ann Intern Med 1993; 119:482-486.

69. Wallace RJ Jr. The clinical presentation, diagnosis, and therapy of cutaneous and pulmonary infections due to the rapidly growing mycobacteria, M. fortuitum and $M$. chelonae. Clin Chest Med 1989; 10:419-429.

\section{Uncorrected Proof Copy}

